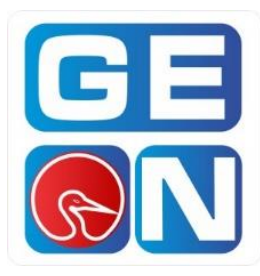

Revista GEON (Gestión, Organizaciones y Negocios.) ISSN: 2346-3910 en línea

revistageon@unillanos.edu.co

Universidad de los Llanos

Colombia

Mondelo Villaseñor, Mónicai; Gamboa Santiago, Roberto Javier;

Menchaca Rodríguez, Rocíoii; Sánchez Márquez, Juan Antonio iii.

Gestión de calidad de la Escuela de Nivel Medio

Superior ENMS de León

Revista GEON, Vol. 6, No. 1, 2019

Pág. 10-22

Disponible en: $\underline{\text { https://doi.org/10.22579/23463910.37 }}$

\footnotetext{
' $\mathrm{https://orcid.org/0000-0002-7925-3005}$

ii https://orcid.org/0000-0002-2007-8011

iii https://orcid.org/0000-0001-9619-8901
}

Esta publicación se encuentra bajo licencia: Creative Commons

ReconocimientoNoComercialSinObraDerivada 4.0 Internacional
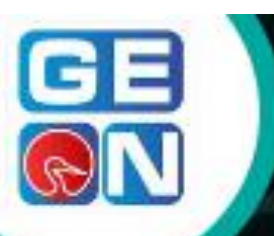

La alta dirección debe estar en una mejora continua en los procesos misionales y administrativos, con la finalidad de tener mejores niveles de competitividad 99 


\title{
Gestión de Calidad de la Escuela de Nivel Medio Superior ENMS de León
}

\section{Quality Management of the Higher Secondary School ENMS de León}

\author{
Mónica Móndelo Villaseñor \\ m.mondelo@ugto.mx
}

Maestra en Gestión y Desarrollo, Universidad de Guanajuato, México, @MonicaMondelo1

\section{Roberto Javier Gamboa Santiago}

rgamboa@ugto.mx

Dr. Ciencias del desarrollo humano, Universidad de Guanajuato, México

\section{Rocio Menchaca Rodríguez \\ r.menchaca@ugto.mx}

Dra. en Pedagogía, Universidad de Guanajuato, México

\section{Juan Antonio Sánchez Márquez \\ r.menchaca@ugto.mx}

Dr. en Ciencias en Ingeniería Química, Universidad de Guanajuato, México

Revista GEON Vol. 6 No 1 enero - junio 2019

ISSN 2346-3910 en línea http://revistageon.unillanos.edu.co/index.php/geon/article/view/37 


\section{Resumen}

La Norma Internacional ISO 10014:2006 "Gestión de la CalidadDirectrices para la obtención de beneficios financieros y económicos", tiene como propósito que la alta dirección de las organizaciones se autoevaluación sobre estos beneficios derivados de la aplicación de la gestión de la calidad.

En el presente trabajo se estableció la obtención de beneficios al interior de la ENMSL aplicando los ocho principios de gestión de la calidad. Se fijaron dos objetivos, el primero relacionado con la medición del nivel de grado de madurez de la ENMSL relacionados al cumplimiento de los ocho principios; y el segundo, la recomendación de métodos y herramientas para la obtención de beneficios. La metodología utilizada aplica un cuestionario de ocho principios de calidad y tres preguntas referenciadas.

El estudio se realizó al personal docente, administrativo y directivo de la institución, obteniéndose el nivel de madurez, su representación gráfica, la priorización de las oportunidades de mejora, el análisis de los resultados de las encuestas y los resultados del nivel de competitividad de la institución.

El nivel de madurez se localiza entre cero (0) a cinco (5). El principio "participación del personal" se posiciona con el menor puntaje (3.5), y el "relación mutuamente beneficiosa con el proveedor" ocupa la posición más alta (4.8). El personal es la esencia de una organización. Se debe contar con personas motivadas, comprometidas en la organización que generen innovación y creatividad al promover los objetivos de la organización siendo responsables de su propio desempeño y participando en la mejora continua.

Palabras claves: Beneficios económicos, Beneficios financieros, Principio de gestión.

\section{Abstract}

The International Standard ISO 10014: 2006 "Quality ManagementGuidelines for obtaining financial and economic benefits", has as purpose that the top management of the organizations self-assessment on these benefits derived from the application of quality management.

In the present work, the obtaining of benefits within the ENMSL was established by applying the eight principles of quality management. Two objectives were set, the first related to the measurement of the degree of 
maturity level of the ENMSL related to compliance with the eight principles; and the second, the recommendation of methods and tools for obtaining benefits. The methodology used applies a questionnaire of eight quality principles and three questions referenced.

The study was carried out to the teaching, administrative and directive personnel of the institution, obtaining the level of maturity, its graphic representation, the prioritization of opportunities for improvement, the analysis of the results of the surveys and the results of the level of competitiveness of the institution.

The level of maturity is between zero (0) to five (5). The principle "staff participation" is positioned with the lowest score (3.5), and the "mutually beneficial relationship with the provider" occupies the highest position (4.8). The staff is the essence of an organization. There must be motivated, committed people in the organization that generate innovation and creativity when promoting the objectives of the organization, being responsible for their own performance and participating in continuous improvement.

\section{Keywords:}

Economic benefits, Financial benefits, Principle of management.

\section{Introducción}

Dentro del plan de trabajo de la investigación a realizar en la ENMSL, se establecerá la obtención de beneficios financieros y económicos en la institución, mediante la aplicación eficaz de los ocho principios de gestión de la calidad, teniendo como propósito principal el de proporcionar a la alta dirección de la escuela, los resultados del nivel de competitividad en la aplicación de la metodología de los ocho principios de la gestión de la calidad.

La Norma Internacional ISO 10014:2006 Gestión de la calidad Directrices para la obtención de beneficios financieros y económicos, tiene como propósito que la alta dirección de las organizaciones que la utilicen, lleven a efecto una autoevaluación sobre los beneficios económicos $\mathrm{y}$ financieros derivados de la aplicación de los principios de gestión de la calidad, así mismo complementa a la norma internacional ISO 9004:2009 Gestión para el éxito sostenido de una organización - Enfoque de gestión de la calidad.

Se fijarán dos objetivos para la investigación, una la medición del nivel de grado de madurez de la ENMSL en relación al cumplimiento de los ocho principios de gestión de la calidad, y otra es la recomendación de métodos y herramientas para la obtención de beneficios financieros y económicos de la ENMSL. 
Las tareas que se llevaron a efecto con los actores (maestros, administrativos y directivos) consistirán en conocer y entender los cinco niveles de madurez, aplicación de las encuestas de autoevaluación tanto inicial como exhaustivas, obtención del nivel de madurez y su representación gráfica mediante el diagrama o carta radar, priorización de las oportunidades de mejora, análisis de los resultados de las encuestas y resultados del nivel de competitividad de la institución. Por último, la obtención de conclusiones y recomendaciones.

Para llevar a efecto el proyecto, se utilizará la metodología proporcionada por la norma internacional ISO 10014:2006 de Gestión de la calidad - Directrices para la obtención de beneficios financieros $\mathrm{y}$ económicos.

La ENMSL, durante los últimos años ha incursionado en los sistemas de calidad de gestión, mediante el diseño y desarrollo e implementación y mantenimiento de la norma internacional ISO 9001:2008, lo cual ha permitido a la institución la obtención de los registros de certificados No. MX08/19389 en ISO 9001:2008 y No. GTO-ISO-001-2009 en GTO-2000 otorgados por los organismos certificadores Societe Generale de Surveillance (SGS) y el Instituto Guanajuato para la Calidad y la Competitividad, respectivamente.

\section{Contexto teórico}

Planteamiento del Problema y Justificación. Los ocho principios de la gestión de la calidad forman parte de los fundamentos de las normas de gestión de la calidad, los cuales fueron elaborados por el Comité Técnico ISO/TC 176 Aseguramiento de la Calidad de la International Organization for Standarization (ISO).

La alta dirección de las instituciones puede utilizar los principios de gestión, como un marco de referencia o directriz para mejorar el desempeño y lograr los objetivos de las empresas o instituciones.

Como uno de los esfuerzos para elevar el nivel de competitividad del sector educativo medio superior, la Escuela de Nivel Medio Superior de León (ENMSL) en los últimos años ha implementado y mantenido el Sistema de Gestión de la Calidad (SGC) ISO 9001:2008 y de Calidad Total mediante el Premio Metropolitano León por la Calidad (PMLC). Teniendo las plataformas anteriores se decidió realizar la presente investigación con el propósito de medir el grado de madurez de la institución en relación al cumplimiento de los principios de gestión, soportado por la metodología que brinda el modelo de la norma internacional "ISO 10014:2006 Gestión de la calidad - Directrices para la obtención de beneficios financieros y económicos".

La ENMSL cuenta con un nivel aceptable de cumplimiento de madurez en referencia a los ocho principios de gestión de la calidad, así mismo se requiere realizar actividades de seguimiento y control en los procesos académicos y administrativos para alcanzar niveles de clase mundial.

La finalidad de la directriz ISO 10014:2006, es la de proporcionar a la alta 
dirección la información que necesita para facilitar la aplicación eficaz de los principios de gestión y la de seleccionar los métodos y herramientas que posibiliten el éxito sostenible de la ENMSL.

Lo anterior permite a la alta dirección evaluar los requisitos, planificar las actividades, asignar los recursos apropiados, implementar acciones de mejora continua y medir los resultados con el fin de determinar su eficacia, así mismo tomar decisiones informadas, ya sea en relación con la definición de estrategias comerciales, el desarrollo de un producto nuevo o la ejecución de acuerdos financieros.

El éxito de la integración de los principios de gestión depende de la aplicación del principio de gestión denominado enfoque basado en procesos y la metodología Planificar-HacerVerificar-Actuar (PHVA).

Al día de hoy no se tiene conocimiento de que alguna otra Institución Educativa haya realizado investigación relacionando la ISO 9004:2009 y la ISO 10014:2006, específicamente en el nivel medio superior. $\mathrm{Y}$ en la Universidad de Guanajuato se tiene implementado un ISO 9001:2008 certificando con éste los Servicios Administrativos que ofrece la Institución Educativa. Generalmente, la ISO 9004:2009 se utiliza de manera constante en la Industria Privada debido a que se hace referencia a beneficios económicos y financieros para lograr el éxito sostenido de una organización.

Objetivo General. Medir el grado de madurez de la institución en relación al cumplimiento de los ocho principios de gestión, soportado por la metodología que brinda el modelo de la norma internacional "ISO 10014:2006 Gestión de la calidad Directrices para la obtención de beneficios financieros y económicos".

Objetivo Secundario. Recomendar los métodos y herramientas para la obtención de beneficios financieros $\mathrm{y}$ económicos de la ENMSL.

Hipótesis y Preguntas de investigación:

¿La ENMSL como institución que cuenta con un registro de certificación en la norma internacional ISO 9001, cuenta con un nivel de madurez aceptable en relación a la aplicación de los ocho principios de gestión de la calidad?

¿Qué métodos y herramientas se pueden recomendar a la alta dirección de la ENMSL, para incrementar los niveles de madurez de los principios de gestión de la calidad que obtengan niveles no aceptables?

\section{Materiales y métodos}

Los conceptos básicos establecidos en este trabajo de investigación se encuentran referenciados en la norma internacional ISO 10014:2006 de Gestión de la calidad - Directrices para la obtención de beneficios financieros y económicos, e ISO 9004:2009 Gestión para el éxito sostenido de una organización - Enfoque de gestión de la calidad.

Beneficios económicos. Se obtienen a través de la gestión eficaz de los recursos y la implementación de procesos aplicables para la mejora del valor y salud general de la organización. 
Beneficios financieros. Es el resultado de la mejora de la organización expresada de forma monetaria, y se obtiene mediante prácticas de gestión de la rentabilidad en la organización.

Éxito sostenido. Resultado de la capacidad de una organización para lograr y mantener sus objetivos a largo plazo.

Entorno de la organización. Combinación de factores y de condiciones internas y externas que pueden afectar al logro de los objetivos de una organización y a su comportamiento hacia las partes interesadas.

Actualmente no se tiene conocimiento de que el modelo utilizado en esta investigación se encuentre establecido en el sector educativo, específicamente en el nivel medio superior.

Los ochos principios de gestión de la calidad constituyen la base de las normas de sistemas de gestión de la calidad de la familia de normas ISO 9000.

A continuación, se presenta la descripción, beneficios y acciones en la implementación de los principios, teniendo como marco conceptual los fundamentos de los sistemas de gestión de la calidad, contenido en el anexo B de la norma internacional ISO 9004:2009 Gestión para el éxito sostenido de una organización - Enfoque de gestión de la calidad.

\begin{tabular}{|c|c|c|}
\hline Descripción: & Beneficios: & Aplicación: \\
\hline \begin{tabular}{l}
\multicolumn{2}{c}{ Las } \\
organizaciones \\
dependen de sus \\
clientes y por lo \\
tanto deberían \\
comprender las \\
necesidades actuales \\
y futuras de los \\
clientes, satisfacer \\
los requisitos y \\
esforzarse \\
exceder en \\
expectativas de los \\
clientes.
\end{tabular} & $\begin{array}{l}\text { - Aumento de los } \\
\text { ingresos y de la cuota de } \\
\text { mercado obtenido } \\
\text { mediante respuestas } \\
\text { flexibles y rápidas a las } \\
\text { oportunidades del } \\
\text { mercado. } \\
\text { - Aumento de la eficacia } \\
\text { en la utilización de los } \\
\text { recursos de la } \\
\text { organización para } \\
\text { mejorar la satisfacción } \\
\text { del cliente. } \\
\text { Mejora de la fidelidad } \\
\text { del cliente que conduce } \\
\text { a la continuidad del } \\
\text { negocio. }\end{array}$ & $\begin{array}{l}\text { 1. Investigar y comprender las } \\
\text { necesidades y expectativas del } \\
\text { cliente. } \\
\text { 2. Asegurarse de que los objetivos } \\
\text { de la organización están } \\
\text { relacionados con las necesidades } \\
\text { y expectativas del cliente. } \\
\text { 3. Comunicar las necesidades y } \\
\text { expectativas del cliente a través } \\
\text { de la organización. } \\
\text { 4. Medir la satisfacción del cliente } \\
\text { y actuar de acuerdo a los } \\
\text { resultados. } \\
\text { 5. Gestionar de manera sistemática } \\
\text { las relaciones con el cliente. }\end{array}$ \\
\hline
\end{tabular}

Fuente: Anexo B, ISO 9004:2009. 
Tabla No.9 Método de investigación de beneficios económicos y financieros.

\begin{tabular}{|c|c|}
\hline Etapas o fases: & Actividades o tareas: \\
\hline \multirow{5}{*}{$\begin{array}{l}\text { Pasos } \\
\text { autoevaluación para lograr } \\
\text { los elementos de entrada. }\end{array}$} & Descripción de los niveles de madurez. \\
\hline & Cuestionario para la autoevaluación inicial. \\
\hline & Cuestionario para la autoevaluación exhaustiva. \\
\hline & Diagrama o carta RADAR. \\
\hline & Oportunidad de mejora - priorización-. \\
\hline \multirow[b]{3}{*}{ Entrada. } & Resultados de la autoevaluación inicial. \\
\hline & Resultados de la autoevaluación exhaustiva. \\
\hline & $\begin{array}{l}\text { Matriz de nivel de madurez autoevaluación inicial } \\
\text { y exhaustiva }\end{array}$ \\
\hline Proceso: metodología & \multirow[t]{2}{*}{ Ciclo de mejora continua. } \\
\hline $\begin{array}{l}\text { PHVA -métodos } \quad \mathrm{y} \\
\text { herramientas-. }\end{array}$ & \\
\hline $\begin{array}{l}\text { Resultados (salida): } \\
\text { Beneficios económicos y } \\
\text { financieros }\end{array}$ & Conclusiones. \\
\hline
\end{tabular}

Tabla No. 1 Principio: Enfoque al cliente.

Fuente: Elaboración propia (2017-2018).

\section{Resultados}

La representación gráfica muestra el modelo genérico del proceso global para la obtención de beneficios financieros y económicos.

Se señala el flujo de seguimiento y control a seguir en cuatro momentos, en los cuales se establece como enfoque basado en procesos, los pasos previos a seguir donde se obtiene la información que servirá de insumo para la entrada de los resultados de las autoevaluación inicial y exhaustiva y como proceso de transformación el ciclo de mejora continua establecido en la metodología PHVA en cada uno de los ocho principios de gestión, así como los métodos y herramientas a utilizar en los mismos y por último como resultado o salida del proceso los beneficios económicos y financieros esperados en la institución.

Aplicación del cuestionario para la autoevaluación inicial.

Para llevar a efecto la aplicación de ambas encuestas, tanto inicial como exhaustiva, el personal académico y administrativo participantes como actores en la aplicación de los cuestionarios, recibieron un curso de formación para el conocimiento y entendimiento de los cinco niveles y descripción de los cinco niveles 
de madurez, así como de las preguntas contenidas en el cuestionario inicial, establecidos en la norma directriz utilizada como metodología en la investigación.

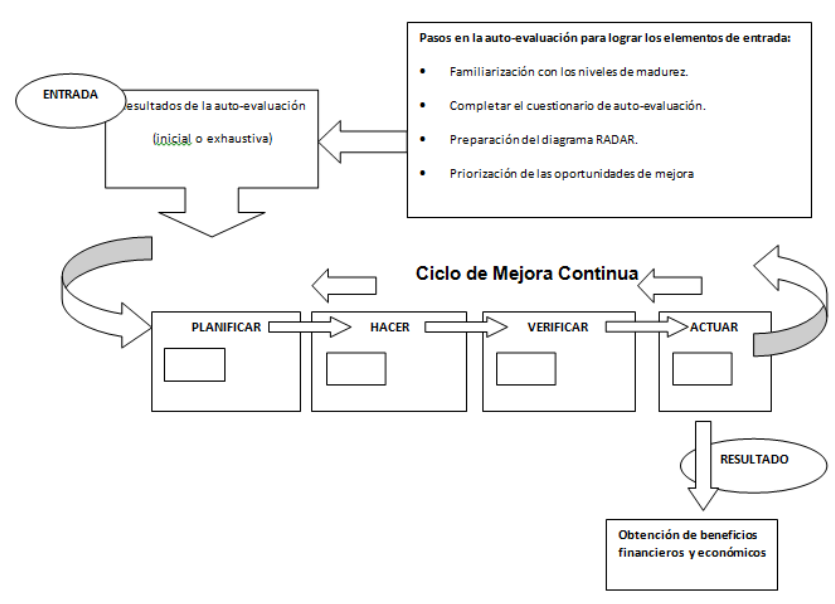

Figura No. 1 - Representación genérica del proceso global para la obtención de beneficios financieros y económicos. Fuente: ISO 10014:2006 Gestión de la calidad -

Directrices para la obtención de beneficios financieros y económicos.

En cada nivel de madurez, se describe con precisión el contenido para su entendimiento, así como la relación entre el nivel de madurez en número absoluto del número uno al cinco, así como su correspondencia en número relativo o porcentual del número porcentual del cero al ciento por ciento.

El cuestionario para la autoevaluación inicial está compuesto por los ocho principios de gestión de la calidad como títulos y tres preguntas referenciadas en relación a los mismos, de tal modo que en total fueron veinte y cuatro preguntas contestadas.

Una vez contestados los cuestionarios, se procesaron en una base de datos electrónica, con la finalidad de obtener el gráfico de figura o diagrama radar y mostrar de manera visual el comportamiento de las diferentes percepciones en relación al nivel de madurez que ocupa en el rango de cero a cinco de los principios de gestión, tal como se muestra en la figura No. 2 Gráfico de radar - principios de gestión, encuestas inicial; en la cual se aprecia que el menor puntaje con 3.5 pertenece al principio de gestión "participación del personal", aunque tiene un comportamiento de un punto por encima de la media (2.5), le falta un punto y medio para obtener el máximo nivel de madurez que es de cinco.

Sí utilizáramos el paradigma de Wilfredo Pareto 80/20, en el cuál considera que resolviendo el $20 \%$ de las causas de los problemas en la organización, pueden coadyuvar a resolver el restante $80 \%$, se tendría que intervenir en cuatro de los ocho principios, tales como el de participación del personal (nivel 3.5), enfoque al cliente, enfoque de sistema para la gestión y mejora continua (nivel 3.6 respectivamente).

Las mejores evaluaciones fueron los principios de gestión, enfoque basados en procesos (3.8), liderazgos y enfoque basa en hechos para la toma de decisiones (4.5 respectivamente) y relación mutuamente beneficiosa con el proveedor (4.8 la más alta). Pareciera que de manera absoluta el nivel presentado de las autoevaluaciones reflejan un grado satisfactorio de los principios de gestión en la ENMSL; analizando el cuadro No. 2, Matriz de nivel de madurez - relación absoluta vs. porcentual -, encuesta inicial; desde el punto de vista de números relativos o porcentual, los cinco principios de gestión que ocupan el nivel de madurez (3) representan entre el $58 \%$ al $63 \%$ de 
nivel de un cien por ciento y solamente los tres restantes principios de gestión que ocupan el nivel de madurez (4) representan entre el $80 \%$ al $90 \%$ de nivel de un cien por ciento. Por lo anterior tenemos escasamente un punto de pase, para no estar reprobados.

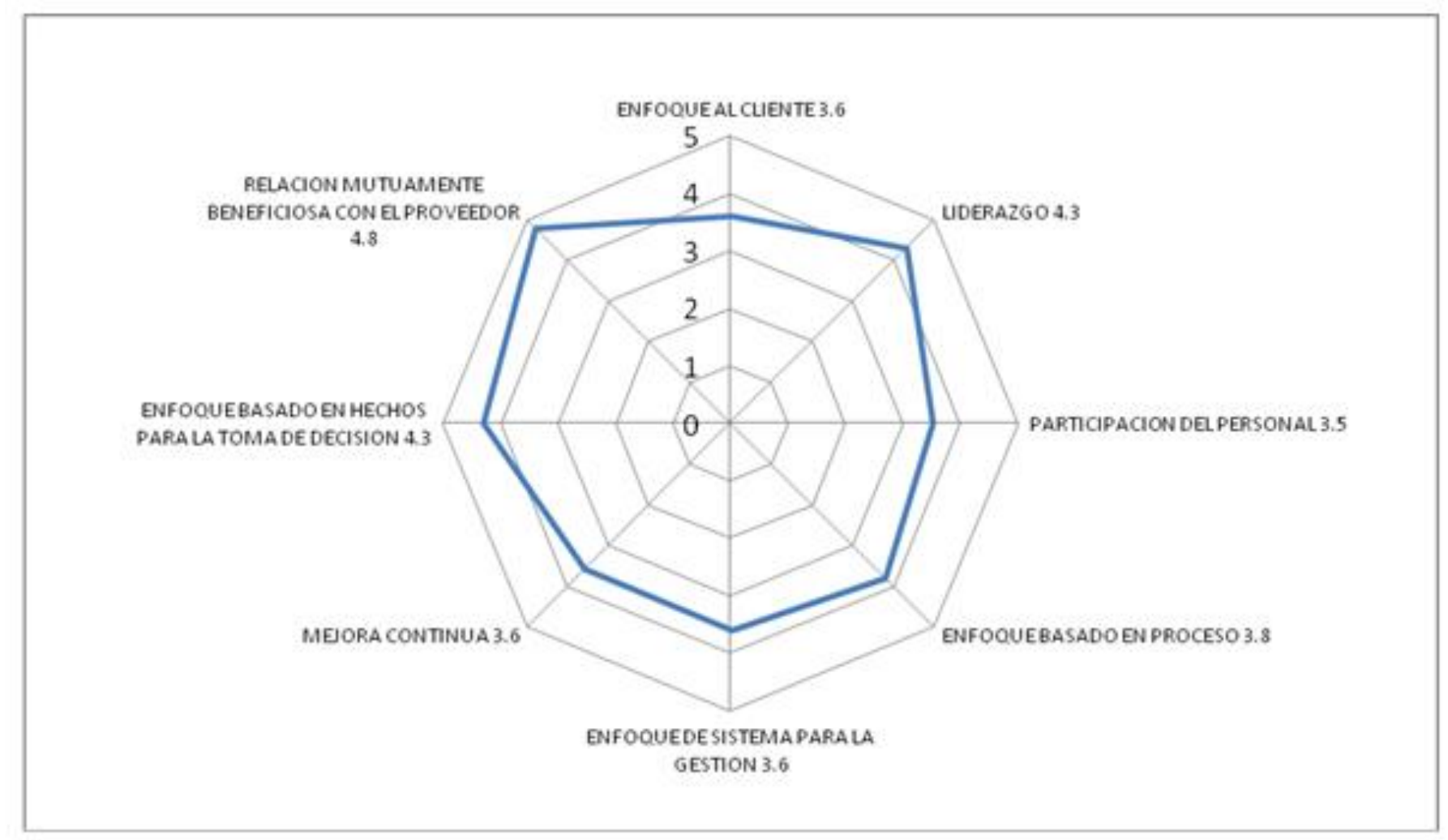

Figura No. 2 - Gráfico Radar - Principios de gestión, encuesta inicial -.

Fuente: Elaboración propia, en base a encuesta inicial, 2017-2018.

Tabla No. 10 Matriz de nivel de madurez - relación absoluta vs. porcentual-, encuesta inicial.

\begin{tabular}{|c|c|c|c|c|c|c|c|c|c|}
\hline $\begin{array}{l}\text { Nivel de } \\
\text { madurez }\end{array}$ & Descripción & \multicolumn{8}{|c|}{ Principios de gestión } \\
\hline $\begin{array}{c}\text { No. } \\
\text { Absolutos }\end{array}$ & $\begin{array}{l}\text { No. } \\
\text { Relativos }\end{array}$ & $\begin{array}{c}\text { Enfoque } \\
\text { al } \\
\text { cliente }\end{array}$ & Liderazgo & $\begin{array}{c}\text { Participación } \\
\text { del } \\
\text { personal }\end{array}$ & $\begin{array}{c}\text { Enfoque } \\
\text { basado } \\
\text { en } \\
\text { procesos }\end{array}$ & $\begin{array}{l}\text { Enfoque } \\
\text { de } \\
\text { sistema } \\
\text { para } \\
\text { la gestión }\end{array}$ & $\begin{array}{l}\text { Mejora } \\
\text { continua }\end{array}$ & $\begin{array}{c}\text { Enfoque } \\
\text { basado en } \\
\text { hechos para } \\
\text { la toma de } \\
\text { decisiones }\end{array}$ & $\begin{array}{c}\text { Relaciones } \\
\text { mutuamente } \\
\text { beneficiosas } \\
\text { con el } \\
\text { proveedor }\end{array}$ \\
\hline 1 & $0 \%$ & & & & & & & & \\
\hline 2 & $25 \%$ & & & & & & & & \\
\hline \multirow{2}{*}{3} & \multirow{2}{*}{$50 \%$} & 3.6 & & 3.5 & 3.8 & 3.6 & 3.6 & & \\
\hline & & $60 \%$ & & $58.30 \%$ & $63.30 \%$ & $60 \%$ & $60 \%$ & & \\
\hline \multirow{2}{*}{4} & \multirow{2}{*}{$75 \%$} & & 4.3 & & & & & 4.3 & 4.8 \\
\hline & & & $80.62 \%$ & & & & & $80.62 \%$ & $90 \%$ \\
\hline \multirow{2}{*}{5} & \multirow{2}{*}{$100 \%$} & & & & & & & & \\
\hline & & & & & & & & & \\
\hline
\end{tabular}

Fuente: Elaboración propia, en base a encuesta inicial, 2017-2018. 
Aplicación del cuestionario para la autoevaluación exhaustiva.

Los diagramas o cartas RADAR proporcionan una visión gráfica del nivel o estado de madurez de la ENMSL en referencia a los ocho principios de gestión, así mismo, ilustra el progreso continuo de la institución.

Las Matrices de nivel de madurez relación absoluta vs. porcentual - de las encuestas, tanto inicial como exhaustiva, muestran un análisis más preciso en relación al desempeño alcanzado en la utilización de los ochos principios de gestión de la calidad en la ENMSL.

En un segundo momento y teniendo como premisa la formación en referencia a los niveles de madurez y preguntas de los cuestionarios de autoevaluación exhaustiva, se aplicó la encuesta exhaustiva, la cual contiene 76 preguntas relacionados a cada uno de los ocho principios de gestión.

Nuevamente los principios de gestión "participación del personal" y "enfoque al cliente" presentaron el menor puntaje (3.3 y 3.4 respectivamente), así mismo presentaron un ajuste a la baja con respecto a la autoevaluación inicial.

El único principio de gestión que permaneció en el nivel de madurez (4) fue el "liderazgo", mientras que los principios de gestión "Enfoque basado en hechos para la toma de decisiones" y "Relaciones mutuamente beneficiosa con el proveedor", bajaron del nivel de madurez (4) al nivel (3) respectivamente.

En general en ambas encuestas inicial y exhaustiva, la percepción del personal académico y administrativo en referencia a la utilización de los ocho principios de gestión de la calidad en la ENMSL, se ubican en el nivel de madurez (3) y solamente el que marca una tendencia a permanecer en el nivel (4) es el principio de gestión "Liderazgo".

La alta dirección de la institución debería aplicar el principio de gestión de la calidad "Enfoque basado en hechos para la toma de decisiones", en referencia a los ocho principios de gestión, con la finalidad de realizar actividades que permitan ir creciendo en los niveles de madurez del (3) hacia el (5).

Algunos resultados que puede obtener la ENMSL de la aplicación de los principios de gestión son, entre otros, aumento de la competitividad, mejora de la retención y lealtad de los clientes, optimización de los recursos disponibles, aumento de la responsabilidad de los empleados, mejora del capital intelectual, optimización de la eficacia y eficiencia de los procesos, sustentabilidad. 


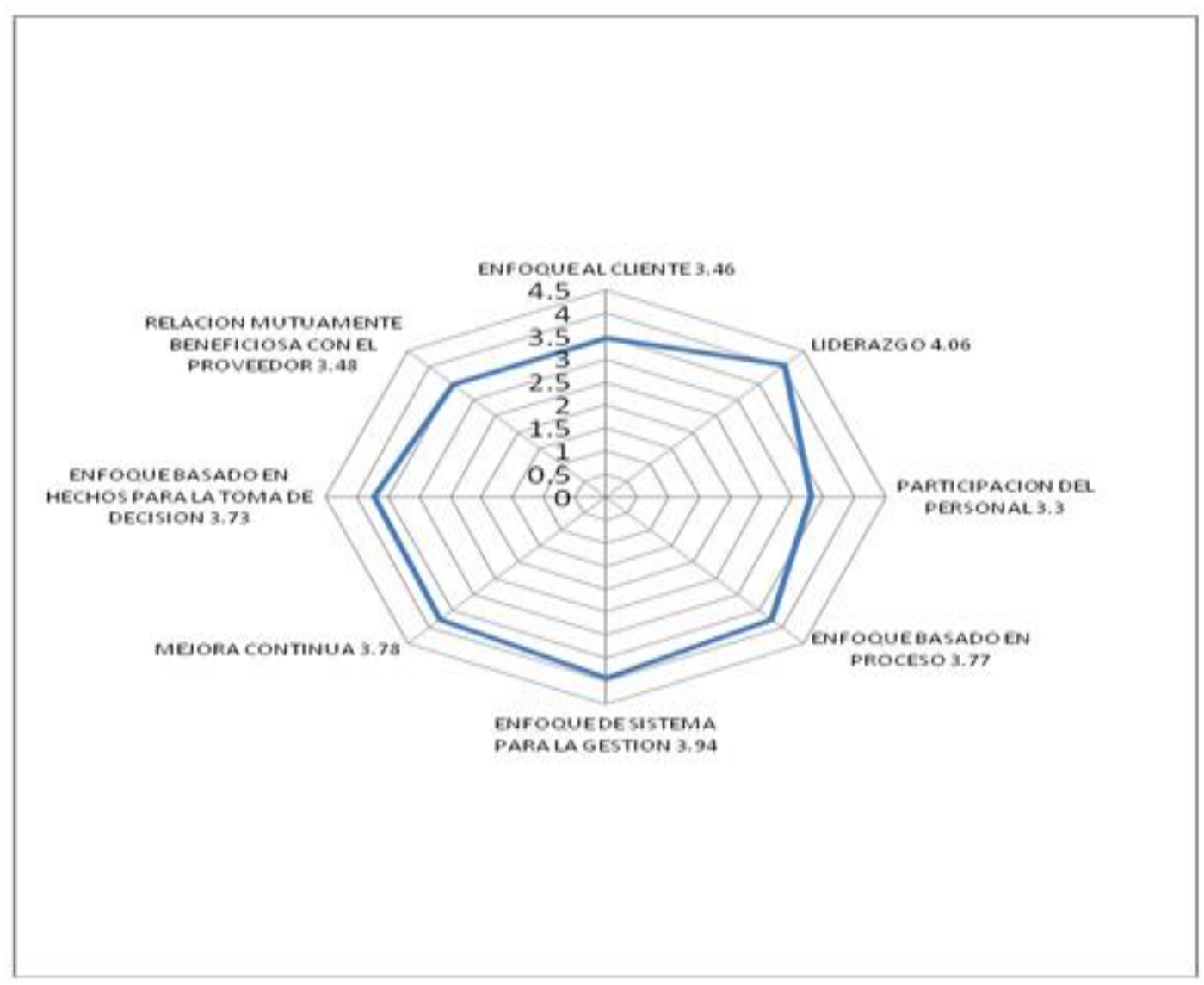

Figura No. 3 - Gráfico Radar - Principios de gestión, encuesta exhaustiva -. Fuente: Elaboración propia, en base a encuesta exhaustiva, 2017-2018.

Tabla No.11 Matriz nivel de madurez-relación absoluta vs. Porcentual-encuesta exhaustiva.

\begin{tabular}{|c|c|c|c|c|c|c|c|c|c|}
\hline $\begin{array}{l}\text { Nivel de } \\
\text { Madurez }\end{array}$ & Descripción & \multicolumn{8}{|c|}{ Principios de gestión } \\
\hline $\begin{array}{c}\text { No. } \\
\text { Absolutos }\end{array}$ & $\begin{array}{c}\text { No. } \\
\text { Relativos }\end{array}$ & $\begin{array}{c}\text { Enfoque } \\
\text { al } \\
\text { cliente }\end{array}$ & Liderazgo & $\begin{array}{c}\text { Participación } \\
\text { del } \\
\text { personal }\end{array}$ & $\begin{array}{c}\text { Enfoque } \\
\text { basado } \\
\text { en } \\
\text { procesos }\end{array}$ & $\begin{array}{c}\text { Enfoque } \\
\text { de } \\
\text { sistema } \\
\text { para } \\
\text { la gestión }\end{array}$ & $\begin{array}{c}\text { Mejora } \\
\text { continua }\end{array}$ & $\begin{array}{c}\text { Enfoque } \\
\text { basado en } \\
\text { hechos para } \\
\text { la toma de } \\
\text { decisiones }\end{array}$ & $\begin{array}{c}\text { Relaciones } \\
\text { mutuamente } \\
\text { beneficiosas } \\
\text { con el } \\
\text { proveedor }\end{array}$ \\
\hline 1 & $0 \%$ & & & & & & & & \\
\hline \multirow{2}{*}{2} & \multirow{2}{*}{$25 \%$} & & & & & & & & \\
\hline & & & & & & & & & \\
\hline \multirow{2}{*}{3} & \multirow{2}{*}{$50 \%$} & 3.46 & & 3.3 & 3.77 & 3.94 & 3.78 & 3.73 & 3.48 \\
\hline & & $58 \%$ & & $55.00 \%$ & $62.83 \%$ & $66 \%$ & $63 \%$ & $62.16 \%$ & $58 \%$ \\
\hline \multirow{2}{*}{4} & \multirow{2}{*}{$75 \%$} & & 4.06 & & & & & & \\
\hline & & & $76.13 \%$ & & & & & & \\
\hline \multirow{2}{*}{5} & \multirow{2}{*}{$100 \%$} & & & & & & & & \\
\hline & & & & & & & & & \\
\hline
\end{tabular}

Fuente: Elaboración propia, en base a datos de la encuesta exhaustiva, 2017-2018. 


\section{Conclusiones}

Al término de la presente investigación, se puede observar que se cumplió con el objetivo de esta, realizando la medición del grado de madurez de los ocho principios de gestión de la calidad en la ENMSL, así como en el cumplimiento de las metas y tareas planteadas.

En términos absolutos el nivel de madurez de la ENMSL en referencia a la aplicación de los ocho principios de gestión de la calidad presenta una madurez por encima de la media, mientras que en términos relativos 0 porcentuales escasamente presenta un $60 \%$ de nivel de crecimiento.

Los resultados obtenidos en la presente investigación, permitirá a la alta dirección de la ENMSL, continuar con la mejora continua en los procesos académicos y administrativos, con la finalidad de tener mejores niveles de competitividad en el sector de la educación. Así mismo, se reconoce que los resultados obtenidos tienen como base el desarrollo de una cultura de calidad que la institución ha permeado en los últimos años.

Algunos de los métodos $\mathrm{y}$ herramientas que pueden ser utilizados para los principios de gestión de la calidad que obtuvieron menor nivel de madurez en la presente investigación son cultura de negocio abierta, planificación de la sucesión, cuadro de mando integral, estudios comparativos con las mejores prácticas, despliegue de la función de calidad, desarrollo por competencias, entre otros.
Se recomienda continuar el seguimiento y medición de la presente, en un segundo momento para este año, con la finalidad de contrastar los resultados y tomar acciones de mejora.

\section{Referencias}

Torralba Flores, A., Pérez Paredes, A., \& Martínez Ángeles, D. (2017). Fracaso Emprendedor, como experiencia de aprendizaje para empresarios del Municipio de Puebla. Revista GEON (Gestión, Organizaciones Y Negocios), 4(2), 25-33. Recuperado a partir de http://revistageon.unillanos.edu.co/index. $\mathrm{php/geon/article/view/21}$

Central Secretariat of ISO (2005). ISO 9000:2005, Quality management systems - Fundamentals and vocabulary. ISO. Switzerland.

Central Secretariat of ISO (2006). ISO 10014:2006, Quality management Guidelines for realizing financial and economic benefits. ISO. Switzerland.

Central Secretariat of ISO (2007). IWA2:2007, Quality management systems - Guidelines for the application of ISO 9001:2000 in education. ISO. Switzerland.

Central Secretariat of ISO (2008). ISO 9001:2008, Quality management systems - Requirements. ISO. Switzerland.

Central Secretariat of ISO (2008). ISO/TC 176/SC 2/N 524R. Introduction and support package: Guidance on ISO 9001:2008 Sub-Clause 1.2 “Application”. 
Central Secretariat of ISO (2008). ISO/TC 176/SC 2/N 525R2. Introduction and support package: Guidance on the documentation requirements of ISO 9001:2008.

Central Secretariat of ISO (2008). ISO/TC 176/SC 2/N 526R2. Introduction and support package: Guidance on the terminology used in ISO 9001 and ISO 9004.

Central Secretariat of ISO (2008). ISO/TC 176/SC 2/N 544R3. Introduction and support package: Guidance on the concept and use of the process approach for management systems.

Central Secretariat of ISO (2008). ISO/TC 176/SC 2/N 630R3. Introduction and support package: Guidance on "Outsource process".

Central Secretariat of ISO (2008). ISO/TC 176/SC 2/N 836. Introduction and support package: Implementing guidance for ISO 9001:2008.

Central Secretariat of ISO (2008). ISO/TC 176/SC 2. Support the publication of ISO 9001:2008 And the Revision of ISO 9004. FAQs on 9001:2008.

Central Secretariat of ISO (2009). ISO 9004:2009, Managing for the sustained success of an organization - A quality management approach. ISO. Switzerland. 